Competing interests None declared.

REFERENCE

1. Novelli M. Dataset for the Histopathological Reporting of Gastric Carcinoma. Standards and Datasets for Reporting Cancers. 2nd edn. The Royal College of

\section{OC-105 IMPACT OF AJCC 7TH EDITION TNM STAGING ON A HISTORICAL OESOPHAGO-GASTRIC CANCER RESECTION DATASET}

doi:10.1136/gutjnl-2012-302514a.105

${ }^{1} \mathrm{~A}$ M Reece-Smith, ' M P John,* ${ }^{2} \mathrm{Z}$ Chaudry, 'S L Parsons. 'Department of Oesophago-gastric surgery, Nottingham University NHS trust, Nottingham, UK; ${ }^{2}$ Department of Histopathology, Nottingham University NHS trust, Nottingham, UK

Introduction The revised AJCC $^{1}$ TNM staging systems for oesophageal and gastric cancer were published in 2010 and have been adopted in many units since that time. However, such changes in staging system can cause problems in comparing recent datasets to historical data as stage matching will no longer be consistent. We aimed to review historical data to determine the impact of updated scoring systems on our dataset, firstly to determine the proportion of patients changing stage groups and secondly to determine if survival was different between new and old systems.

Methods A database of gastro-oesophageal resections has historically accumulated patients with operative stage recorded using 6th edition TNM classification. With the help of a specialist pathologist these cases were reassessed to determine the revised TNM according to the 7 th edition. The survival of matched stage disease was compared using Kaplan-Meier analysis with log-rank test for statistical significance.

Results In a cohort of 358 patients 50 patients (14.0\%) changed stage. Twenty-four to a lower stage and 26 to higher stages as detailed in the Abstract OC-105 table 1 below. Stage 2 and 3 contained sufficient patients for survival analysis. Median survival was not reached for stage 2 in TNM6 and was 37.9 months in TNM7 ( $p=0.651$ ). In stage 3 the survival was 16.7 months in TNM6 and 17.3 months using TNM7 ( $p=0.786)$. Survival was not significantly different between editions.

Conclusion Fourteen percent of patients change stage using updated criteria. However, the impact of these changes on the median survival of patients in specified stage groups is small. Re-evaluating historical patient data will not greatly advise clinicians or patients regarding their prognosis but this data does help in the comparison of historical publications to current data.

Competing interests None declared.

\section{REFERENCE}

1. Ann Surg Oncol 2010;17:1721-4. Pathologists. 2007.

\section{OC-106 SERONEGATIVE VILLOUS ATROPHY - COELIAC DISEASE JUST HALF THE TIME?}

doi:10.1136/gutjnl-2012-302514a.106

${ }^{1} \mathrm{I}$ Aziz, ${ }^{1}{ }^{1} \mathrm{~K}$ E Evans, ${ }^{2} \mathrm{~S} S \mathrm{C}$ Cross, ${ }^{1} \mathrm{D}$ S Sanders. ${ }^{1}$ Department of Gastroenterology, Royal Hallamshire Hospital, Sheffield, UK; ${ }^{2}$ Department of Histopathology, Royal Hallamshire Hospital, Sheffield, UK

Introduction Villous atrophy (VA) in the presence of a positive coeliac serology (endomysial [EMA] and tissue transglutaminase [tTG] antibodies) is highly predictive of coeliac disease (CD). However, diagnostic challenges may arise in those where VA is associated with a negative coeliac serology.

Aims To study the aetiology of seronegative VA.

Methods One hundred patients presenting with seronegative VA (both EMA negative and tTG normal range) were prospectively and systematically investigated for $\mathrm{CD}$ and other known associations of VA. The history, immunoglobulins and histopathology were initially reviewed, followed if necessary by a combination of HLA typing, gluten challenge, repeat coeliac serology \& duodenal biopsies, and exclusion of infection/inflammatory bowel disease.

Results 59 women, 41 men, age range 16-92 years, median age 49 years. CD was present in $44 \%$ of cases, followed by gastrointestinal infections (18\%), severe duodenitis (4\%), crohn's disease $(3 \%)$, tuberculosis $(2 \%)$, HIV $(2 \%)$, drugs $(2 \%)$ and haematological disorders $(2 \%)$. In $23 \%$ an association was not found, although the majority normalised their small bowel histology on repeat biopsies $(17 / 23,74 \%)$. Reviewing the case with a specialist gastrointestinal (GI) histopathologist played an important role in up to $9 \%$ of cases, in terms of identifying a cause or help tailoring further investigations. All coeliac patients tested for HLA status were as expected positive for DQ2 or DQ8 haplotypes (n 37), in comparison to 22/47 $(47 \%)$ non-coeliacs $(\mathrm{p}<0.0001)$. A non-coeliac diagnosis was significantly more common in asian/african patients or those with clinical symptoms of abdominal pain, weight loss or nausea \& vomiting $(p<0.05)$. However, there was no difference in age, gender, baseline biochemistry or haematological blood tests between the two groups. Conclusion Close collaboration with a specialist GI histopathologist is useful in cases of seronegative VA. A cause will be found in $77 \%$ of patients, with CD accounting for almost half of all cases. In the $23 \%$ with no apparent cause, reassuringly $74 \%$ normalised their histology. The minority with persisting VA of unknown cause remain under active follow-up. The prevalence of a positive HLA in cases deemed to be non-coeliacs was greater than that of $25 \%$ as accepted for the general population. Many of these cases were due to gastrointestinal infections or an unknown cause, and subsequently normalised their histology (18/22). A threshold model for CD has recently been proposed and these patients may still belong to this disease spectrum-further work looking for intestinal antibody deposits in this group may shed light on this possibility.

Competing interests None declared.

\section{OC-107 MORPHOMETRIC ANALYSIS OF FIBROSIS AND INFLAMMATORY ACTIVITY IN RECURRENT HCV INFECTION POST LIVER TRANSPLANTATION}

Abstract OC-105 Table 1

doi:10.1136/gutjnl-2012-302514a.107

\begin{tabular}{lrr}
\hline Stage change TNM6 to TNM7 & $\mathbf{N}=$ & $\%$ \\
\hline Stage 4 to stage 3 & 14 & 3.9 \\
Stage 2 to stage 1 & 10 & 2.8 \\
Stage 1 to stage 2 & 13 & 3.6 \\
Stage 2 to stage 3 & 13 & 3.6 \\
No change & 308 & 74.8 \\
Total & 358 & 100.0 \\
\hline
\end{tabular}

${ }^{1,2} \mathrm{~S} M$ Noory, ${ }^{*}{ }^{1,2} \mathrm{R}$ Hajhosseiny, ${ }^{1,2} \mathrm{G}$ Noory, ${ }^{2} \mathrm{D}$ Joshi, ${ }^{2} \mathrm{~A}$ Quaglia. ${ }^{1}$ School of Medicine, King's College London University, London, UK; ${ }^{2}$ Institute of Liver Studies, King's College Hospital, London, UK

Introduction Hepatitis $\mathrm{C}$ virus (HCV) recurrence post liver transplantation (LT) is universal. Ishak score (IS) is widely used in the histological assessment of recurrent HCV. Smooth muscle actin (SMA) expression is a marker of hepatic stellate cells (HSC), 